\title{
Photonic simulation software tools for education
}

\section{Jason Taylor, Stoyan Tanev}

Jason Taylor, Stoyan Tanev, "Photonic simulation software tools for education," Proc. SPIE 9665, Tenth International Topical Meeting on Education and Training in Optics and Photonics, 96651N (3 June 2007); doi: 10.1117/12.2207668

SDIE Event: Tenth International Topical Meeting on Education and Training in Optics and Photonics, 2007, Ottawa, Ontario, Canada 


\title{
Photonic Simulation Software Tools for Education
}

\author{
Jason Taylor \\ Optiwave Systems Inc. \\ 7 Capella Court, Ottawa, ON, Canada, K2E 7X1 \\ Dr. Stoyan Tanev \\ Department of Systems and Computer Engineering \\ Carleton University, 1125 Colonel By Drive, Ottawa, ON, Canada, K1S $5 B 6$
}

\begin{abstract}
A solid education in optical devices and optical communication systems must include an understanding of the basic building blocks of optical devices and networks as well as the interplay between them. Software vendors, such as Optiwave Systems Inc., provide free as well as for-purchase software tools that can be used in classroom and computer labs as an educational aid. This paper examines the role software simulation tools play in the education of students studying optical communication and related disciplines. The different techniques to employ photonic simulation software in classroom lectures, computer labs and graduate research are discussed.
\end{abstract}

\section{Introduction}

Despite volatile market conditions, optical communication systems continue to evolve at a rapid pace. In recent years, the focus of industry research has shifted from long haul to metro to fiber to the home networks [1]. These different networks require very different solutions. Many new optical components have been developed and continue to be developed to address these requirements. It is difficult if not impossible for educational institutes to keep student laboratories equipped with a complete inventory of optical components required to demonstrate realistic optical networks. They could, however, develop design and simulation capabilities that may provide a similar and even higher value for educators and students of photonic device and network courses as well as of fundamental optics and electromagnetic courses in general.

Education in optical systems requires knowledge of several disciplines including signal analysis, optics, and electronics. Physical scales range from nanometer device features to thousands of kilometers of optical fiber links. For a student studying optical system design, designing an optical link is about generating the signal transmitting and propagating it over a given distance while maintaining a minimum signal quality. The signal quality depends on many parameters and device characteristics of the electric pulse generators, light sources, modulators, optical channel (typically fiber), photodetectors and receiver electronics (see for example Agrawal [2]). The function of each one of these devices is based on optical and electrical phenomena which can be quite subtle to understand.

This paper will focus on how photonic simulation software (PSS) such as OptiSystem and OptiFDTD can be used to enhance education of phonic devices and optical communication. This paper is organized in the following way: section II provides some background of PSS is. In section III, the general methods for using PSS as an educational tool are discussed. Section IV contains some representative examples of how PSS is used in education. Finally, section V contains a conclusion.

\section{Photonic simulation software (PSS)}

In the domain of PSS, there are many tools from nano/micro-scale component simulation software such as OptiFDTD (finite-difference time-domain simulator), to large scale system software such as OptiSystem (optical communication system simulation software). In between, there are many other specialized tools for designing waveguides, optical fiber, optical gratings, bulk optics components etc.

Low level software such as OptiFDTD, simulate the propagation of optical fields through nano- to micro-scaled devices by directly solving Maxwell's equations numerically [3]. Waveguide software such as OptiBPM, is a specialized software tool used to simulate waveguides and other similar optical devices which have light propagation predominantly in one direction over large distances. OptiBPM is based on the beam propagation method (BPM). In essence, the BPM is a semi-analytical technique that solves an approximation of the wave equation [4]. OptiSystem is a simulation package that can design, test and optimize virtually any type of optical link in the physical layer of a wide range of optical networks [5]. It is based on large collection of realistic models for components and sub-systems.

Tenth International Topical Meeting on Education and Training in Optics and Photonics, edited by Marc Nantel, Proc. of SPIE Vol. 9665, 96651N · @ 2007 SPIE, OSA, IEEE, ICO doi: $10.1117 / 12.2207668$ 


\section{Methodology}

There are 3 basic levels that PSS can introduced as an educational aid:

1. Lecture examples and visualization,

2. Student assignments and labs and

3. Graduate and post-graduate research.

The most basic use of PSS in education is to supplement classroom lectures. Most commercially available tools provide excellent visualization capabilities for input (system and structure layout) as well as output (graphs, tables etc.). This technique can be used even for students who may not have proficient computer skills since there is no requirement for the student to run the software. It can be used to introduce fundamental concepts of Maxwell's equations to and high level designs of optical systems.

Currently, the greatest increase in the use of PSS in education is in computer labs. In this scenario, professors create assignments and labs for students to solve using PSS. Traditionally, student assignments were often limited to problems that could be solved analytically in a few pages of handwritten calculations. While this is a very important component of learning optics, the limitation severely restricts the amount of problems that can be assigned. Even when the problems can be solved analytically, complex mathematics required for the solution can often obfuscate the underlying physics or engineering concepts that are the purpose of the assignment. Professional PSS have user interfaces that are very closely related to real world problems. In OptiFDTD the user, uses a graphical interface to draw the structure to be simulated. In OptiSystem, the user arranges and connects icons that represent real devices in a clearly visual way.

There are many benefits of using PSS in computer labs. Students have the options to perform virtual experiments instantly. Simulations provide an excellent way to build an intuitive understanding of the physical effects that occur. They provide instant feedback on "what if?" scenarios. This allows students to follow their own curiosity. Computer simulations in a lab environment provide a complimentary educational tool that lies between theory and physical experiments. On one hand, simulations can easily handle a large number of practical problems that are not possible to solve analytically. On the other hand, many more structures and system can be simulated than is practical to construct for real world experiments. In addition to this, computer simulations also provide instant visualization of many more physical qualities than can be obtained easily in experiments. As an example, users can view plots of the electric field inside a waveguide at any point in time; something that can not be done with a real device.

For now, the most common use of PSS in education is at the graduate and post-graduate levels for research. For many years, simulation software has been recognized as an important tool for the design and development of new devices and systems. Device and system researchers can concentrate on concepts and design without spending effort to create the numerical software themselves.

Experience with professional quality simulation tools is an asset in itself. Professional software tools are used by researchers in telecommunication and other high tech companies all around the world. Students that have experience with such software during their formal education have clear advantage over students that do not.

\section{Examples}

In this section, 2 representative examples are given to illustrate how PSS can be used as a demonstration tool in a classroom environment. The two examples represent opposite ends of the photonic simulation spectrum. In the first example, OptiFDTD is used to demonstrate an important concept of the microscopic vector nature reflected optical waves. The second example demonstrates how a free software, OptiPerformer, can be used in a computer lab tool for an optical communication course.

\section{Vector nature of electromagnetic waves}

Photonic simulation software can be used for the demonstration of fundamental optics phenomena such as simple reflection, refraction, absorption etc. The particular example considered here is based on the OptiFDTD software - a time-domain simulation tool numerically solving Maxwell's equations. OptiFDTD is applied, in parallel with an analytical analysis, [6] to demonstrate the vector nature of the electromagnetic (EM) waves during the reflection of a linearly polarized EM wave from a mirror (Fig. 1, left) positioned in free space. This is a 2D propagation problem in a plane defined by the $\mathrm{Z}$ (longitudinal) 
and X (transverse) axes of a coordinate system (the Y direction is perpendicular to the X-Z plane and points towards the reader). For the sake of simplicity we consider the case where the input optical beam (positioned at $\mathrm{X}=3 \mu \mathrm{m}$ with a Gaussian profile with a half-width of $2 \mu \mathrm{m}$ ) is linearly polarized and there is only one component of the electric field (Ey). Our goal is to provide a visual explanation of the relationship between the direction of EM power flow and the different non-zero components of the electric and magnetic fields. The power flow of an EM wave is described by the time averaged value of the Poynting vector $\mathbf{S}$ which is proportional to the vector product of the magnitude of the electric and magnetic fields: $\mathbf{E} \times \mathbf{H}$. $S_{x}$ is proportional to $\left(E_{y} H_{z}-E_{z} H_{y}\right)$ and $S_{z}$ is proportional to $\left(E_{x} H_{y}-E_{y} H_{x}\right) . S_{y}$ is equal to zero since the propagation is only in the $\mathrm{X}-\mathrm{Z}$ plane. Since we consider a linearly polarized $\mathrm{EM}$ wave ( $\mathrm{E}_{\mathrm{y}}$ field component only), the two non-zero components of the Poynting vector take a simpler form: $S_{x} \sim E_{y} H_{z}$ and $S_{z} \sim E_{y} H_{x}$.
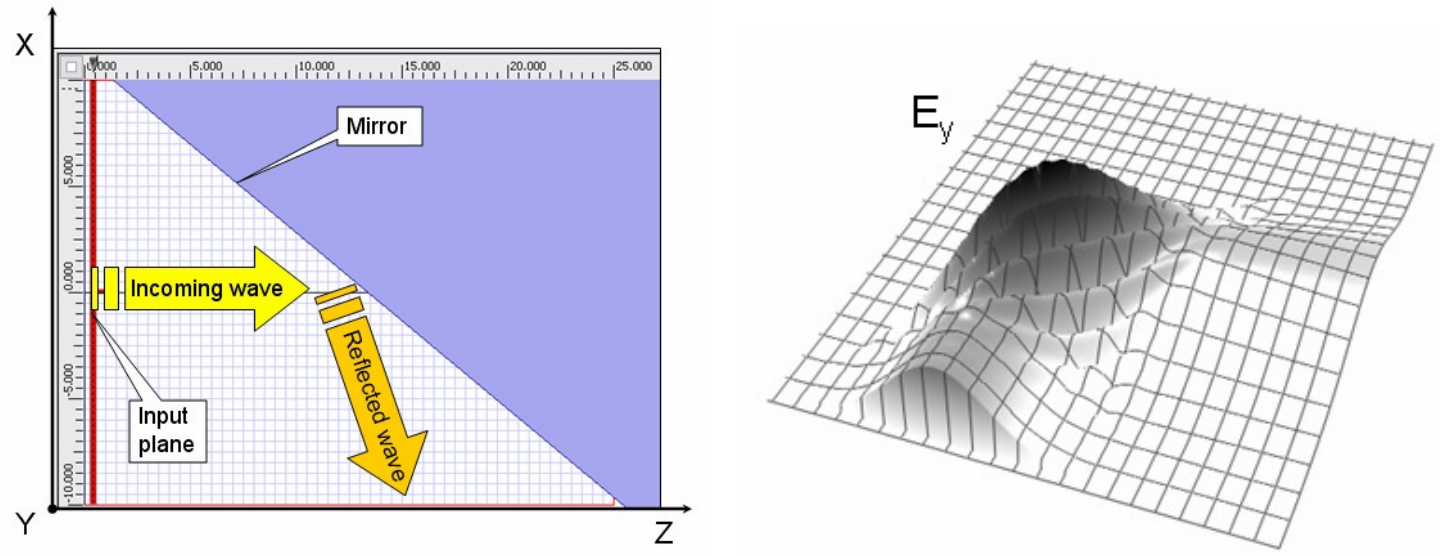

Fig. 1 Schematic representation of the propagation geometry (left) and distribution of the $\mathrm{E}_{\mathrm{y}}$ electric field magnitude (right).
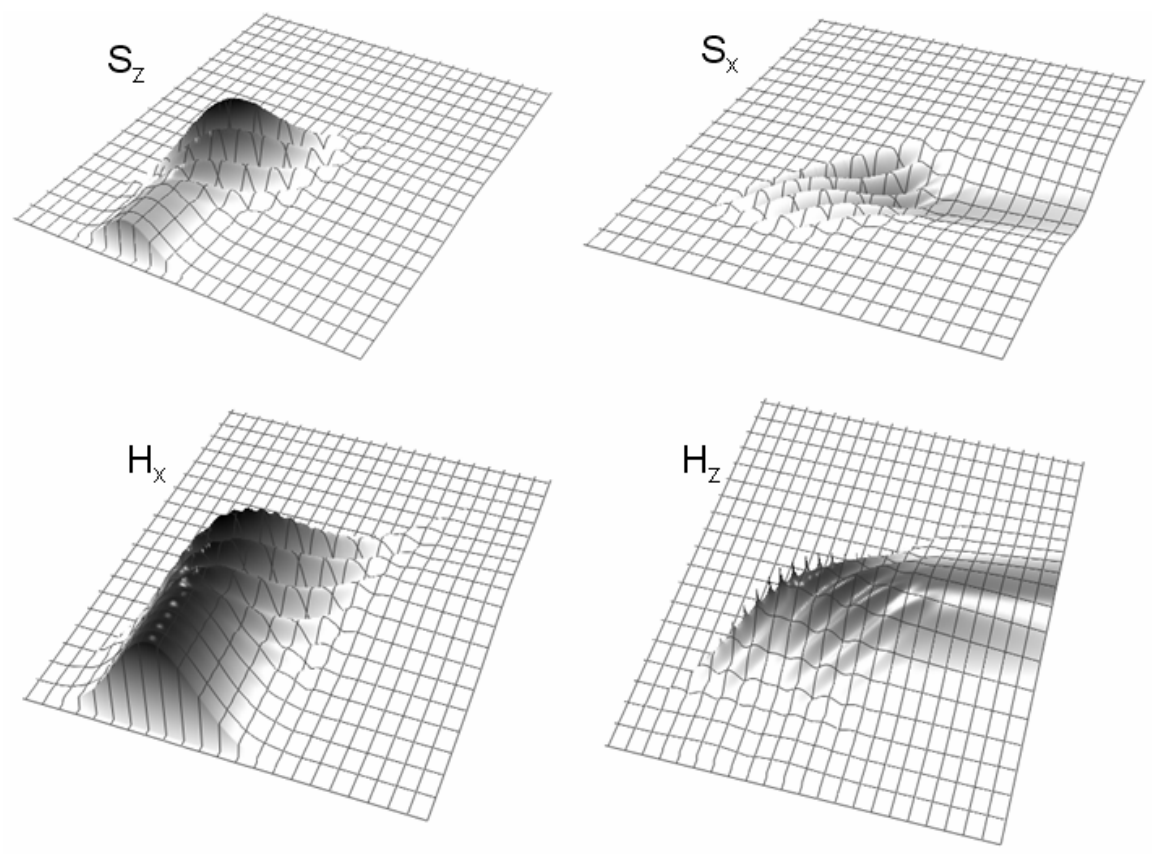

Fig. 2 Visual representation of the distribution of i) the longitudinal $\left(S_{z}\right)$ and transverse $\left(S_{x}\right)$ components of the Poynting vector and ii) the magnitude $\mathrm{H}_{\mathrm{x}}$ and the $\mathrm{H}_{\mathrm{z}}$-fields. The redirection of energy in the X-direction is associated with a transfer of energy from the $\mathrm{H}_{\mathrm{x}}$ to the $\mathrm{H}_{\mathrm{z}}$ component of the magnetic field. 
An examination of Fig. 1 (right) can show that if the incoming wave is propagating initially in the Z-direction (i.e., $S_{x}$ is negligibly small), the mirror will redirect the wave energy predominantly in the perpendicular (X-) direction. Due to the energy conservation law the above mentioned redirection of the energy flow will be associated with a decrease of the magnitude of $\mathrm{S}_{\mathrm{z}}\left(\right.$ or $\left.\mathrm{H}_{\mathrm{x}}\right)$ and increase of the magnitude of $\mathrm{S}_{\mathrm{x}}\left(\mathrm{or}_{\mathrm{z}}\right)$. This transfer of energy can be clearly seen in Fig. 2. It should be pointed out that the distribution of the $S_{\mathrm{x}}$-component of the Poynting vector has negative values. This indicates that the energy flow is in the opposite direction of the $\mathrm{X}$ coordinates axis.

\section{Example 2 Attenuation-Limited Fiber Length}

The next example is based on a lab exercise created by Dr. Warren Koontz [7]. This lab can be downloaded from Optiwave web, including the software, OptiPerformer [8], required to run it. The object of the lab is to calculate the attenuation-limited fiber length based on a power budget equation then to simulate and verify that it meets the performance objectives.

The power budget equation states that the transmitted power minus the receiver sensitivity must be greater than or equal to the sum of the power losses plus the power margin.

$$
P_{T}-S_{R}=A L_{F}+L_{C}+L_{A}+M,
$$

where $\mathrm{P}_{T}$ is the transmitter power, $S_{R}$ is the receiver sensitivity, $A$ is the fiber attenuation, $L_{F}$ is the fiber length, $L_{C}$ is the coupling loss, $\mathrm{L}_{\mathrm{A}}$ is the additional know losses and $\mathrm{M}$ is the power margin.

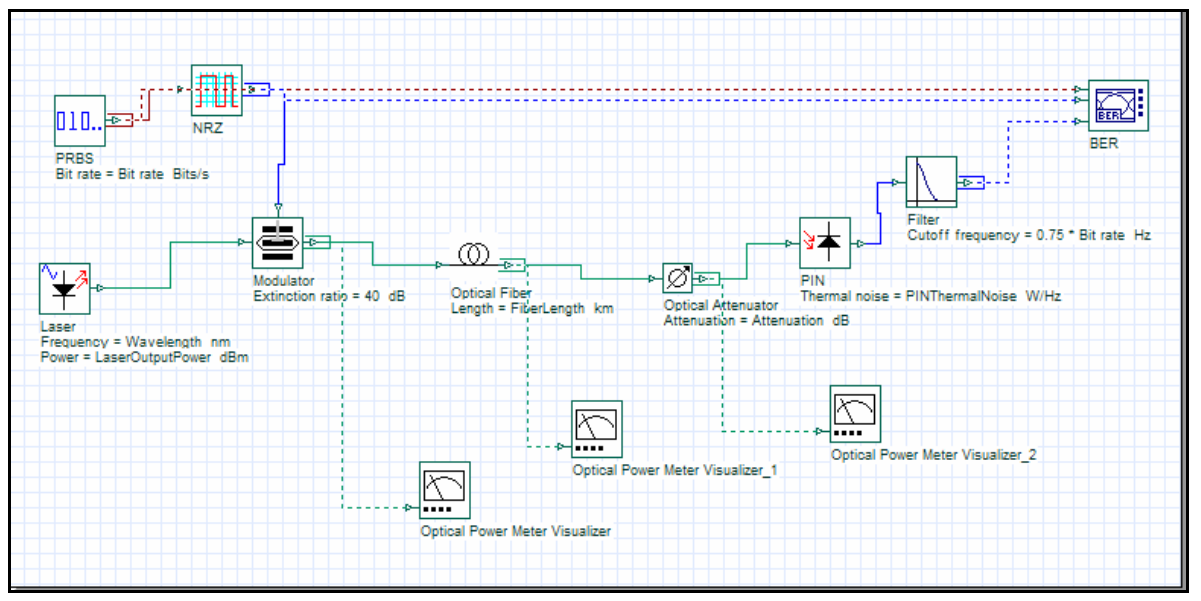

Fig. 3 Screen capture of OptiPerformer. The layout is of a single one channel optical link.

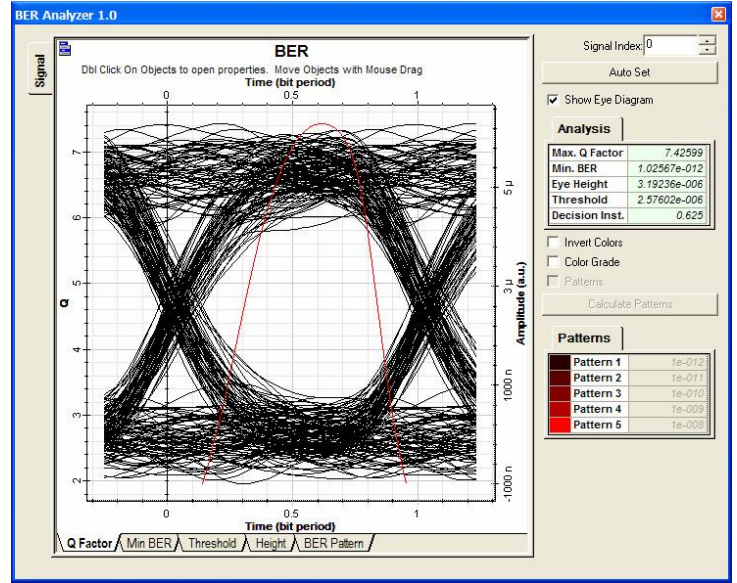

Fig. 4 Screen capture of the BER analyzer. A graph of the Q factor as a function of decision instant is shown with the eye diagram superimposed on it. 
The OptiPerformer layout of the single channel link is shown in Fig. The layout is fixed but the user can modify the laser output, the fiber length, the attenuation coefficient of the fiber, the thermal noise of the PIN detector and the wavelength of the laser. OptiPerformer files are created using, OptiSystem. Much more complex system layouts than this one can be created.

The output of the BER analyzer is shown in Fig. 4. This gives the user visual feedback on the quality of the signal as well as numerical analysis of the quality of the signal. This diagram is calculated for any input parameters the user specifies. In this case, the fiber length was set to $120 \mathrm{~km}$. The resulting maximum Q factor is 7.42 which corresponds to a bit error rate of $1.03 \times 10^{-12}$.

\section{Conclusion}

Photonic simulation software can be a valuable aid in the education of optical device and optical communication systems. It provides a method to move past the sometimes too abstract level of theory to gain insight into physical phenomenon associated with optical devices and systems. It provides more freedom to explore design parameters than analytic calculations and physical experiments. This allows students to develop an intuitive understanding of optics in a rapid way.

\section{References}

[1] Lightwave online article. http://lw.pennnet.com/display_article/289131/13/ARTCL/none/none/US-FTTH-connectionscontinue-to-accelerate

[2] G. Agrawal, Fiber-Optic Communication Systems (John Wiley \& Sons, 1997)

[3] OptiFDTD 7.0: Technical Background and Tutorials, (Optiwave Systems Inc., 2007)

[4] OptiBPM 8.0: Getting Started, (Optiwave Systems Inc., 2007)

[5] OptiSystem 6.0: Getting Started and Tutorials, (Optiwave Systems Inc., 2007)

[6] Eugene Hecht, Optics, Second Edition, Addison-Wesley, Reading, 1989, p. 41.

[7] This and other sample labs can be obtained from Optiwave via a request sent to info@optiwave.com

[8] OptiPerformer can be downloaded from the Optiwave web site http://www.optiwave.com. 